OPEN ACCESS

Edited by:

Dr. Souhaila Al Khodor,

Sidra Medical and Research Center,

Qatar

Reviewed by:

Paul M. Coussens,

Michigan State University, USA

Jianping Xie,

Southwest University, China

*Correspondence:

Xiangmei Zhou

zhouxm@cau.edu.cn

Received: 06 August 2016 Accepted: 28 November 2016 Published: 12 December 2016

Citation:

Cui Y, Zhao D, Sreevatsan S, Liu C, Yang $W$, Song Z, Yang L, Barrow $P$

and Zhou X (2016) Mycobacterium bovis Induces Endoplasmic Reticulum

Stress Mediated-Apoptosis by

Activating IRF3 in a Murine

Macrophage Cell Line.

Front. Cell. Infect. Microbiol. 6:182.

doi: 10.3389/fcimb.2016.00182

\section{Mycobacterium bovis Induces Endoplasmic Reticulum Stress Mediated-Apoptosis by Activating IRF3 in a Murine Macrophage Cell Line}

\author{
Yongyong Cui ${ }^{1}$, Deming Zhao ${ }^{1}$, Srinand Sreevatsan ${ }^{2}$, Chunfa Liu ${ }^{1}$, Wei Yang ${ }^{1}$, \\ Zhiqi Song ${ }^{1}$, Lifeng Yang ${ }^{1}$, Paul Barrow ${ }^{3}$ and Xiangmei Zhou ${ }^{1 *}$ \\ 'State Key Laboratories for Agrobiotechnology, Key Lab of Animal Epidemiology and Zoonosis, Ministry of Agriculture, \\ National Animal Transmissible Spongiform Encephalopathy Laboratory, College of Veterinary Medicine, China Agricultural \\ University, Beijing, China, ${ }^{2}$ Veterinary Population Medicine Department, College of Veterinary Medicine, University of \\ Minnesota, St. Paul, MN, USA, ${ }^{3}$ School of Veterinary Medicine, University of Nottingham, Sutton Bonington, UK
}

Mycobacterium bovis ( $M$. bovis) is highly adapted to macrophages and has developed multiple mechanisms to resist intracellular assaults. However, the host cells in turn deploy a multipronged defense mechanism to control bacterial infection. Endoplasmic reticulum (ER) stress-mediated apoptosis is one such primary defense mechanism. However, the role of interferon regulatory factor 3 (IRF3) between ER stress and apoptosis during $M$. bovis infection is unknown. Here, we demonstrate that $M$. bovis effectively induced apoptosis in murine macrophages. Caspase-12, caspase-9, and caspase-3 were activated over a $48 \mathrm{~h}$ infection period. The splicing of XBP-1 mRNA and the level of phosphorylation of elF2 $\alpha$, indicators of ER stress, significantly increased at early time points after $M$. bovis infection. The expansion of the ER compartment, a morphological hallmark of ER stress, was observed at 6 h. Pre-treatment of Raw 264.7 cells with 4-PBA (an ER stress-inhibitor) reduced the activation of the ER stress indicators, caspase activation and its downstream poly (ADP-ribose) polymerase (PARP) cleavage, phosphorylation of TBK1 and IRF3 and cytoplasmic co-localization of STING and TBK1. M. bovis infection led to the interaction of activated IRF3 and cytoplasmic Bax leading to mitochondrial damage. Role of IRF3 in apoptosis was further confirmed by blocking this molecule with BX-795 that showed significant reduction expression of caspase-8 and caspase-3. Intracellular survival of $M$. bovis increased in response to 4-PBA and BX-795. These findings indicate that STING-TBK1-IRF3 pathway mediates a crosstalk between ER stress and apoptosis during $M$. bovis infection, which can effectively control intracellular bacteria.

Keywords: mycobacterium, M. bovis, ER stress, IRF3, apoptosis 


\section{INTRODUCTION}

It is more than a hundred years since Mycobacterium tuberculosis (Mtb) was first isolated by Robert Koch (Cui et al., 2016). Tuberculosis (TB) remains one of the most serious global diseases affecting humans and animals (WHO, 2014). Mycobacterium bovis ( $M$. bovis), a species belonging to Mycobacterium tuberculosis Complex, is the main cause of TB in cattle, deer and other mammals and it also can infect people by drinking or eating contaminated, unpasteurized dairy products or close contact with infected animals (Arap et al., 2004; Waters and Palmer, 2015). Macrophages are the major cell type infected by Mtb and M. bovis. Consequently, host innate immune mechanisms have co-evolved to better counter mycobacterial infections (Aldwell et al., 1996). Host macrophages also induce an apoptotic signal to control bacterial infection (Rodrigues et al., 2013; Srinivasan et al., 2014). Understanding the mechanism of apoptosis induced by $M$. bovis infection will lead to better delineate host immune responses that could be exploited to control infection.

Endoplasmic reticulum (ER) is not only the major site of folding and transportation of proteins after synthesis, but also the major site of storage of intracellular $\mathrm{Ca}^{2+}$ and synthesis of cholesterol, steroids, and lipids. Recent studies have demonstrated that infection of macrophages by the virulent $\mathrm{H} 37 \mathrm{Rv}$ and attenuated $\mathrm{H} 37 \mathrm{Ra}$ strains results in increases in the amount of rough- (RER) and smooth- (SER) endoplasmic reticulum respectively (Saquib et al., 2015). And mycobacterial infection results in loss of $\mathrm{Ca}^{2+}$ from the ER and an increase in the intracellular redox state which results in accumulation of unfolded or misfolded proteins in the ER resulting in ER stress, characterized by an expansion in the ER compartment (Choi et al., 2010; Verfaillie et al., 2012; Lim et al., 2013, 2015). The host cell responds with unfolded protein response (UPR) which suspends the synthesis of new proteins and thereby reduces accumulation of unfolded or misfolded proteins in the ER to restore normal physiological function (Ron and Walter, 2007). Prolonged and uncontrolled ER stress can lead to activation of a triad of signaling pathways moving the cell toward apoptosis (Tabas and Ron, 2011; Hetz, 2012). Inositol-requiring enzyme 1 (IRE1), protein kinase RNA (PKR)-like ER kinase (PERK), and activating transcription factor 6 (ATF6) are mainly three pathways that participate in ER stress (Rasheva and Domingos, 2009; Sano and Reed, 2013). However, it is unknown whether ER stress is also involved in M. bovis infection and the mechanism of macrophages apoptosis.

It is well-known that stimulator of interferon genes (STING) is located in the outer membrane of the ER. Recent research has documented that ER stress causes the translocation of STING into the cytoplasm, where STING recruits TANK-binding kinase 1 (TBK1) to activate interferon regulatory factor 3 (IRF3) (Liu et al., 2012; Petrasek et al., 2013). Activated IRF3 likely causes the release of cytochrome $\mathrm{c}$ by a variety of mechanisms, leading to the damage of mitochondria and eventually, cell death (Chattopadhyay et al., 2010; Vince and Tschopp, 2010).

Here we show that ER stress of macrophages leads to apoptosis in a caspase-dependent manner during M. bovis infection. And we demonstrate that ER stress-induced apoptosis is mediated by the activation of IRF3, which requires STING and TBK1. Both mechanisms effectively control intracellular M. bovis killing. To our knowledge, this is the first report to investigate ER stress in $M$. bovis infection and it's association with IRF3 release leading to apoptosis.

\section{MATERIALS AND METHODS}

\section{Reagents and Antibodies}

Rabbit monoclonal anti-phospho-eIF2 $\alpha$ (Ser51) antibody, rabbit monoclonal anti-phospho-IRF-3 (Ser396) antibody were acquired from Cell Signaling Technology (Danvers, MA, USA). Rabbit monoclonal anti-TBK1 antibody was purchased from Abcam (Cambridge, UK). Rabbit polyclonal anti-STING antibody, rabbit polyclonal anti-PARP antibody, and rabbit polyclonal anti-tubulin antibody were obtained from Santa Cruz Biotechnology (Santa Cruz, CA, USA). Rabbit polyclonal anti-caspase-3 (p17 specific) antibody, rabbit polyclonal anti-caspase- 8 antibody, rabbit polyclonal anti-caspase-9 antibody, rabbit polyclonal anti-caspase-12 antibody, rabbit polyclonal anti-BAX antibody, and rabbit polyclonal anti-cytochrome $c$ antibody were purchased from Proteintech (Wuhan, China). Rabbit polyclonal anti-VDAC1 was acquired from Sangon Biotech (Shanghai, China). PeroxidaseConjugated Affinipure goat anti-rabbit $\operatorname{IgG}(\mathrm{H}+\mathrm{L})$ (ZB- 5301) and goat anti-mouse $\operatorname{IgG}(\mathrm{H}+\mathrm{L})$ (ZB- 5305), Alexa Fluor 488conjugated Affinipure goat anti-rabbit $\operatorname{IgG}(\mathrm{H}+\mathrm{L})(\mathrm{ZB}-0511)$ were purchased from ZSGB Biotechnology (Beijing, China). Donkey anti-goat IgG/Alexa Fluor 647 was purchased from Biosynthesis Biotechnology (Beijing, China). Tunicamycin was purchased from Fermentek Ltd (Jerusalem, Israel). BX-795 was purchased from Selleckchem (Houston, TX, USA).

\section{Cell Culture}

Murine macrophage Raw 264.7 cell line was obtained from China Infrastructure of Cell Line Resources (Beijing, China), and was maintained in Dulbecco's modified Eagle's medium (HyClone, South Logan, Utah, USA) supplemented with $10 \%$ fetal bovine serum, 1\% L-glutamine (Gibco, Grand Island, NY, USA), penicillin (10,000 units/ml), streptomycin $(10,000 \mu \mathrm{g} / \mathrm{ml})$ (HyClone, South Logan, Utah, USA) at $37^{\circ} \mathrm{C}$ with $5 \% \mathrm{CO}_{2}$.

\section{M. bovis Culture}

$M$. bovis Beijing strain was obtained from China Institute of Veterinary Drug Control (CVCC, China) and was grown in Middlebrook 7H9 liquid medium (BD Biosciences, NY, USA) with $10 \%$ ADC (albumin, dextrose, catalase), and $0.05 \%$ Tween80 and grown to mid-log phase for 1 week at $37^{\circ} \mathrm{C}$. Aliquots were frozen at $-80^{\circ} \mathrm{C}$ until used.

\section{M. bovis Infection and Colony-Forming Unit Assay}

Macrophages were infected in vitro with $M$. bovis at an MOI of 10 and incubated for $3 \mathrm{~h}$ at $37^{\circ} \mathrm{C}$ with $5 \% \mathrm{CO}_{2}$. After allowing $3 \mathrm{~h}$ for phagocytosis, the cells were washed three times with warm PBS to remove extracellular bacteria and then incubated with fresh medium without antibiotics for an additional time. They 
were then lysed in autoclaved distilled water to allow collection of intracellular bacteria. The lysates were plated separately on Middlebrook $7 \mathrm{H} 11$ agar plates and incubated at $37^{\circ} \mathrm{C}$ with $5 \%$ $\mathrm{CO}_{2}$ for 3 weeks. Colony counts were performed in triplicate.

\section{Apoptosis Analysis}

Apoptotic cells were assessed using an Annexin V/propidium iodide (AV/PI) staining kit according to the manufacturer's instructions (KeyGENBioTECH, Nanjing, China). Binding of Annexin V and PI was analyzed by BD FACSCalibur flow cytometer (BD Biosciences, San Jose, CA, USA) with FlowJo software (Tree Star, Ashland, OR, USA).

\section{Transmission Electron Microscopy}

Cells were infected and then washed three times with PBS, trypsinized, fixed in ice-cold $5 \%$ glutaraldehyde in $0.1 \mathrm{M}$ sodium cacodylate buffer $(\mathrm{pH} 7.4)$ at $4^{\circ} \mathrm{C}$ for $15 \mathrm{~min}$, and then centrifuged. Cell pellets were fixed for $4 \mathrm{~h}$. After a complete rinse with sodium cacodylate buffer, the cell pellet was further fixed in $1 \%$ OsO4 in $0.1 \mathrm{M}$ sodium cacodylate buffer on ice for $1 \mathrm{~h}$ and dehydrated with acetone. The cell pellet was embedded in EM-bed 812 resin and polymerized at $60^{\circ} \mathrm{C}$ for $48 \mathrm{~h}$. Ultrathin sections $(70 \mathrm{~nm})$ were obtained on a Leica Ultracut UCT ultramicrotome (Vienna, Austria) and counterstained with uranyl acetate and lead citrate before observation under a fluorescence microscope (Olympus Fluoview, Japan).

\section{RT-PCR Analysis}

Total RNA was extracted using the EASYspin Plus RNA Extraction Kit (Aidlab, Beijing, China). Then total RNA was reverse-transcribed to cDNA using the Maxima First Strand cDNA synthesis kit (Thermo Scientific, Waltham, MA, USA). For determination of XBP-1 splicing, the cDNA product was subjected to 35 cycles of PCR using the forward primer 5'-AAACAGAGTAGCAGCTCAGACTGC$3^{\prime}$ and the reverse primer $5^{\prime}$-TCCTTCTGGGTAGACCTCa TGGGAG-3' which are specific for mouse XBP-1. $\beta$-actin (forward: CCTTCTGACCCATTCCCACC; reverse: GCTTCTTTGCAGCTCCTTCG) was used as an housekeeping control. Products were separated by electrophoresis through a $3 \%$ agarose gel.

\section{Immunofluorescence Microscopy}

For protein localization analysis, Raw 264.7 cells grown on cover slips were washed twice with PBS, fixed by Immunol Staining Fix Solution, blocked $1 \mathrm{~h}$ at room temperature by Immunol Staining Blocking Buffer (Beyotime Biotechnology, Shanghai, China) and then incubated overnight at $4^{\circ} \mathrm{C}$ with the appropriate primary and secondary antibodies. The nuclei were stained with DAPI. Raw 264.7 cells were labeled using anti-phospho-IRF3 antibody, anti-STING antibody, anti-TBK1 antibody, and fluorescein isothiocyanate (FITC)-conjugated donkey anti-goat, Alexa Fluor-conjugated goat anti-rabbit and FITC-conjugated goat anti-rabbit antibodies as the primary and secondary antibodies respectively. Macrophages were finally mounted with glycerin buffer and examined immediately with an OLYMPUS microscope. All experiments were performed on three independent occasions in triplicate at each time.

\section{Western Blot and Co-Immunoprecipitations}

Cellular proteins were extracted with lysis buffer and total protein concentrations were determined with the Bradford assay and $30 \mathrm{mg}$ of protein was separated with SDS-PAGE, followed by electrotransfer to a Immobilon-P Membrane (Millipore, MA, USA). The blots were probed with primary antibodies and secondary antibodies at optimized concentrations. Enhanced chemiluminescence was used as WB detection system. Image J (NIH, Washington, C, USA) was used for quantification of immunoblots. For immunoprecipitation, cell lysates were pre-cleared using protein A/G agarose (Santa Cruz, CA, USA) beads at $4^{\circ} \mathrm{C}$ for $30 \mathrm{~min}$ on a rocker. The beads were removed by centrifugation at $13,200 \mathrm{rpm}$ at $4^{\circ} \mathrm{C}$ for $1 \mathrm{~min}$ and transferred the supernatant to a fresh centrifuge tube. Then protein A/G agarose/sepharose slurry was incubated with anti-IRF3 antibody overnight at $4^{\circ} \mathrm{C}$. Beads were washed 3-4 times with RIPA or NP40 buffer. The agarose/sepharose beats were resuspended in the sample buffer and were boiled for $5 \mathrm{~min}$. The beats were then spinned down and the supernatants were used for SDS-PAGE and western blot analysis.

\section{Isolation of Mitochondria and Cytoplasm}

Isolation of mitochondria and cytoplasm from Raw 264.7 whole cell lysates was performed with the Subcellular Structure Mitochondrial Isolation Kit and the Subcellular Structure Cytoplasmic and Nuclear Isolation Kit respectively according to manufacturer's protocol (Boster, Wuhan, China).

\section{Mitochondrial Transmembrane Potential $\left(\Delta \Psi_{\mathrm{m}}\right)$ Assay}

Mitochondrial transmembrane potential was assessed using the cationic fluorescent indicator JC-1 (Molecular Probes, Eugene, OR, USA), which aggregates in intact mitochondria (red fluorescence) with normal $\Delta \Psi \mathrm{m}$, but remains in the monomeric form in the cytoplasm (green fluorescence) of cells with disrupted mitochondrial membrane. Raw 264.7 cells were incubated in DMEM medium containing $10 \mu \mathrm{M} \mathrm{JC}-1$ at $37^{\circ} \mathrm{C}$ for $15 \mathrm{~min}$, washed with PBS, and then transferred to a clear 24-well plate. JC-1 aggregate fluorescent emission was measured at $583 \mathrm{~nm}$ with an excitation wavelength of $526 \mathrm{~nm}$; and JC-1 monomer fluorescence intensity was measured with excitation and emission wavelengths at 525 and $530 \mathrm{~nm}$. Finally, the cells were mounted with the DakoCytomation fluorescent medium and visualized via fluorescence microscopy (Olympus Fluoview, Japan).

\section{Statistical Analysis}

All assays were performed on three separate occasions in triplicate at each time. Results are expressed as means \pm S.D. All comparisons of data were made using one-way ANOVA followed by post-hoc Tukey's test or Student's $t$-test. SPSS software (version 13.0: SPSS Inc., Chicago, IL, USA), GraphPad Prism 5 
software (La Jolla, CA, USA) and Image J (National Institutes of Health, USA) were used, and $P<0.05$ was considered significant.

\section{RESULTS}

\section{M. bovis Infection Induces Apoptosis}

Apoptosis plays an important role in host defense against mycobacterial infection (Xu et al., 2014; Liu et al., 2015). We first investigated if intracellular M. bovis could induce apoptosis in macrophages. Initial evaluation of MOI to efficiency of phagocytosis in the RAW 264.7 macrophages showed that $98 \%$ of the cells were infected with an MOI of 10 at $24 \mathrm{~h}$. Flow cytometric analysis with Annexin V/propidium iodide (AV/PI) staining was used to quantify cells undergoing apoptosis after $24 \mathrm{~h}$ of infection with M. bovis. As shown in Figures 1A,B, the percentage of early apoptotic and late apoptotic cells increased from $0.7 \%$ in control cells to $9.5 \%$ after $M$. bovis infection. Caspases were activated in M. bovis infected Raw 264.7 cells. Cleavage of caspase-12 and caspase- 9 gradually increased in a time-dependent manner over a $48 \mathrm{~h}$ period, while caspase- 3 started to decrease by $24 \mathrm{~h}$ post infection (pi) (Figures 1C,D). These data indicate that M. bovis induces macrophage apoptosis.

\section{ER Stress Is Induced during M. bovis Infection}

Previous studies have shown that mycobacterium-induced apoptosis is associated with ER stress (Seimon et al., 2010; Sohn et al., 2011). To determine if M. bovis-induced apoptosis was also linked to ER stress, morphology of ER was detected by transmission electron microscopy (TEM). We found expansion of the ER compartment within $6 \mathrm{~h}$ after $M$. bovis infection, a morphological hallmark of ER stress (Figure 2A). Apoptosis of macrophages was significantly decreased when the chemical chaperone 4-phenyl butyric acid (4-PBA), an ER stress inhibitor, was used to treat cells prior to $M$. bovis infection (Figures 1A,B). ER stress triggers apoptosis mainly through the PERK and IRE1 $\alpha$ pathways (Sano and Reed, 2013). To investigate whether these two pathways were activated during $M$. bovis infection in Raw 264.7 cells, we examined the levels of expression of the markers of ER stress. Expression and the splicing of X-box
A

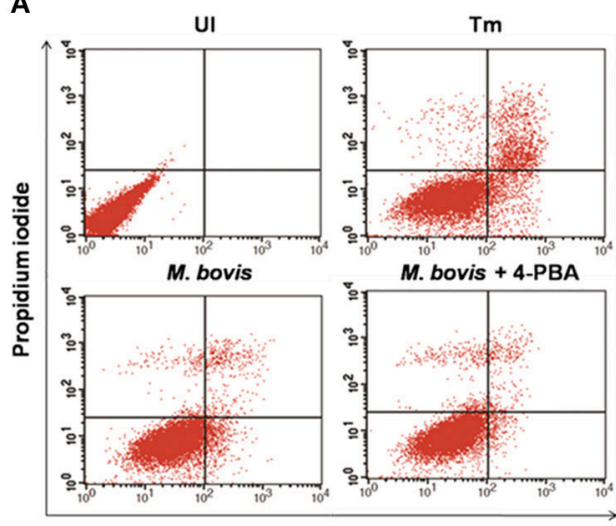

C

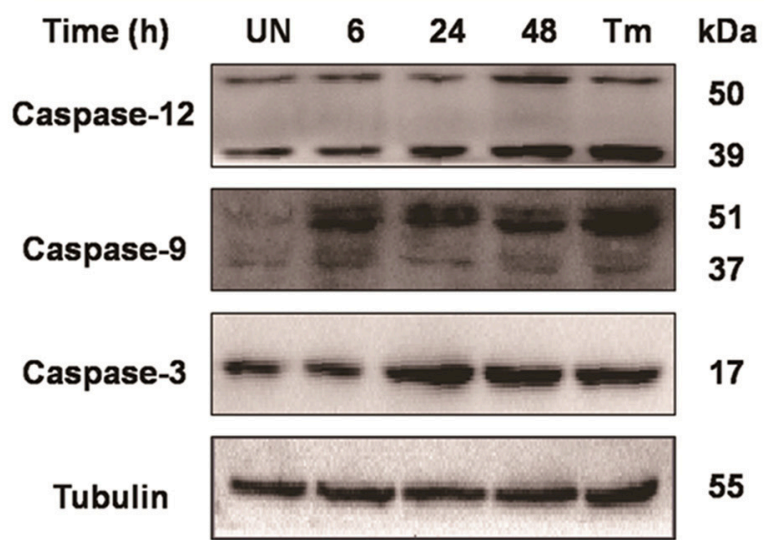

B

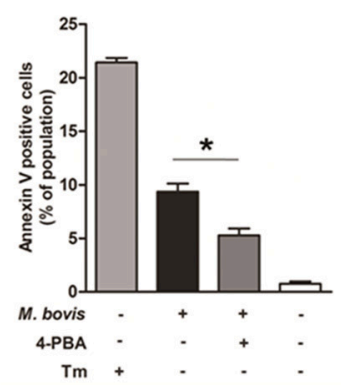

D

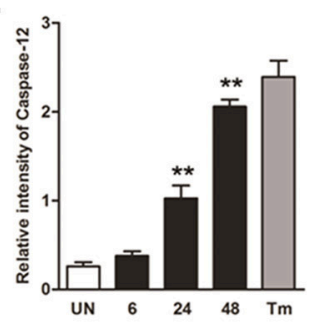

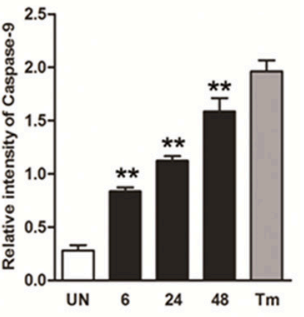

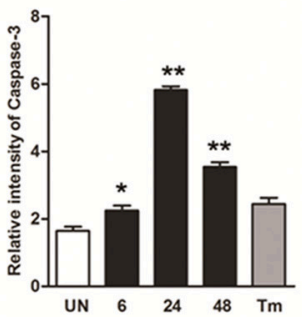

FIGURE 1 | M. bovis infection induces apoptosis and caspase activation. (A) Raw 264.7 cells were screened for induction of apoptosis using Annexin V/PI staining after $24 \mathrm{~h}$ infection with $\mathrm{M}$. bovis at an $\mathrm{MOI}$ of 10 . Tunicamycin $(5 \mu \mathrm{g} / \mathrm{ml})$ was used as a positive control for apoptosis. 4-PBA, an ER stress inhibitor was treated before M. bovis infection in Raw 264.7 cells. After washing and Annexin V/PI staining, cells were analyzed by flow cytometry. (B) Quantitative analysis of the percentage of apoptotic cells (sum of early and late apoptotic cells) from (A), a statistically significant difference ${ }^{*} P<0.05$ ) is observed between 4-PBA pretreated and non-pretreated groups using the two-tailed $t$-test. (C) Raw 264.7 cells were stimulated with $M$. bovis for $24 \mathrm{~h}$ and total cell lysates were subjected to Western blot for cleaved caspase-3 (p17 specific), caspase-9, and caspase-12. (D) Bands corresponding to each protein were quantified, and the intensities of each protein were normalized to the intensity of tubulin. Data are representative of at least three independent experiments, each performed in triplicate with similar results. The asterisks indicate statistically significant differences compared with untreated cells $\left({ }^{\star} P<0.05,{ }^{\star \star} P<0.01\right)$. 
A
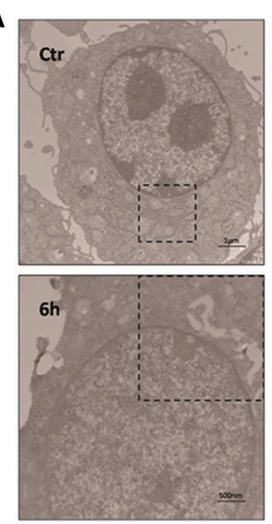

C

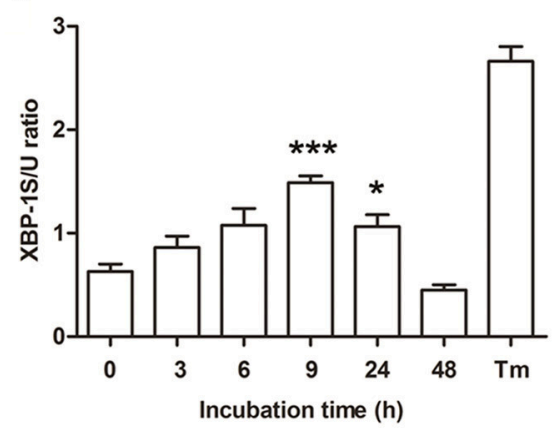

B

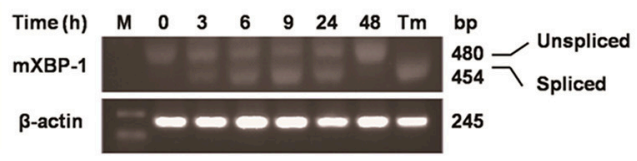

D
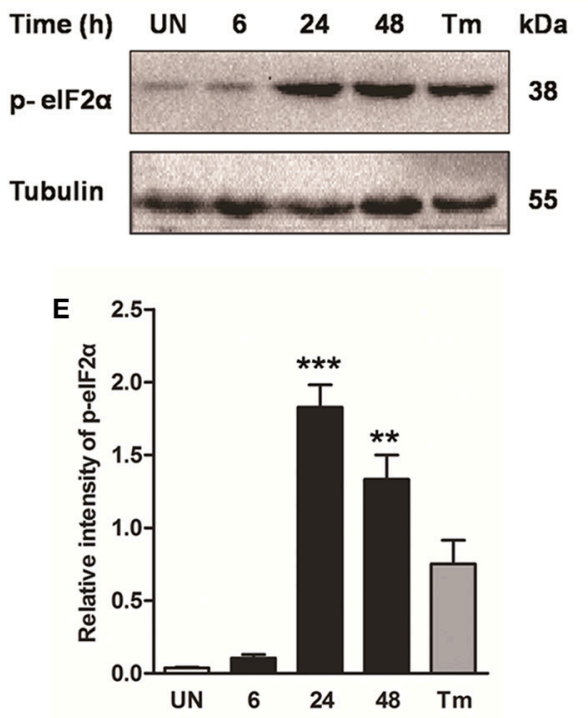

FIGURE 2 | ER stress is involved in M. bovis-mediated apoptosis. Raw 264.7 cells were infected with $M$. bovis at an MOl of 10 , and then incubated for $0-48 \mathrm{~h}$. (A) TEM analysis of Raw 264.7 cells after $M$. bovis infection for $6 \mathrm{~h}$. The perinuclear rough ER regions on the images in the left panel are magnified on the right panels. Arrows indicate ER lamellae before and after infection. (B) XBP-1 mRNA splicing was determined by RT-PCR using specific primers that were used to amplify products of unspliced and spliced mRNA. (C) The results represent the ratio of spliced XBP-1 to intact (or unspliced) XBP-1 (XBP-1S/U ratio). (D) Total cell lysates were subjected to Western blot to identify phosphorylation of elF2 $\alpha$. (E) Bands corresponding to each protein were quantified, and the intensities of each protein were normalized to the intensity of tubulin. Data are representative of at least three independent experiments, each performed in triplicate with similar results. The asterisks indicate significant differences compared with untreated cells $\left({ }^{\star} P<0.05\right.$, ${ }^{\star \star} P<0.01$, ${ }^{\star \star \star} P<0.001$ ).

binding protein 1 (XBP-1) mRNA was markedly increased at $9 \mathrm{~h}$ pi and then gradually decreased until $48 \mathrm{~h}$ pi (Figures $2 \mathrm{~B}, \mathrm{C}$ ). Phosphorylation of eukaryotic translation initiation factor $2 \mathrm{~A}$ $(\mathrm{eIF} 2 \alpha)$ appeared as early as $6 \mathrm{~h}$ pi and reached the peak at $24 \mathrm{~h}$ pi (Figures 2D,E). These results suggest that $M$. bovis interacts with ER and inducing stress.

\section{Effect of ER Stress-Mediated Apoptosis on Intracellular Survival of $M$. bovis}

Based on above studies, we reasoned that $M$. bovis-induced apoptosis was associated to the ER stress pathways (Lim et al., 2011; Choi et al., 2013). Raw 264.7 cells were treated with 4PBA before $M$. bovis infection. Phosphorylation of eIF2 $\alpha$ was significantly attenuated by the treatment with $5 \mathrm{mM} 4$-PBA at $6 \mathrm{~h}$ and $24 \mathrm{~h} \mathrm{pi,compared} \mathrm{to} \mathrm{respective} \mathrm{control} \mathrm{cells} \mathrm{(Figures} \mathrm{3A,B).}$ In response to the treatment with $2.5 \mathrm{mM}$ and $5 \mathrm{mM} 4$ PBA, phosphorylation of eIF $2 \alpha$ and the cleavage of caspase-12 which resides in the ER, caspase- 3 and its downstream cleaved poly (ADP-ribose) polymerase (PARP) were attenuated in a dose-dependent manner at $24 \mathrm{~h}$ pi (Figures 3C,D). Then we posited that ER stress could influence survival of intracellular bacteria. ER stress agonist tunicamycin (Tm) was used prior to $M$. bovis infection. We found that the total numbers of intracellular bacteria were respectively decreased by $44.4 \%$ and $33.8 \%$ compared to macrophages without Tm treatment at $6 \mathrm{~h}$ and $24 \mathrm{~h}$ pi (Figure 3E). We further confirmed that intracellular survival of $M$. bovis was significantly increased by $53.7 \%$ in response to 4 -PBA at $24 \mathrm{~h}$ pi (Figure 3F). These data suggest that M. bovis-induced apoptosis is mediated by ER stress, which can effectively control intracellular mycobacteria.

\section{ER Stress Results in the Phosphorylation and Nuclear Translocation of IRF3}

Previous studies have suggested that IRF3, a transcription factor regulating innate immune responses, was involved in ER stress-mediated apoptosis (Liu et al., 2012; Petrasek et al., 2013; Jin et al., 2015). However, the role of IRF3 in M. bovis infection is unknown. Thus, the phosphorylation of IRF3 was studied during $M$. bovis infection. Our studies show that phosphorylation of IRF3 ensued $M$. bovis infection at $24 \mathrm{~h}$ pi (Figures 4A-D). Nuclear translocation of IRF3 implies that phosphorylation has occurred (Majumdar et al., 2015). 

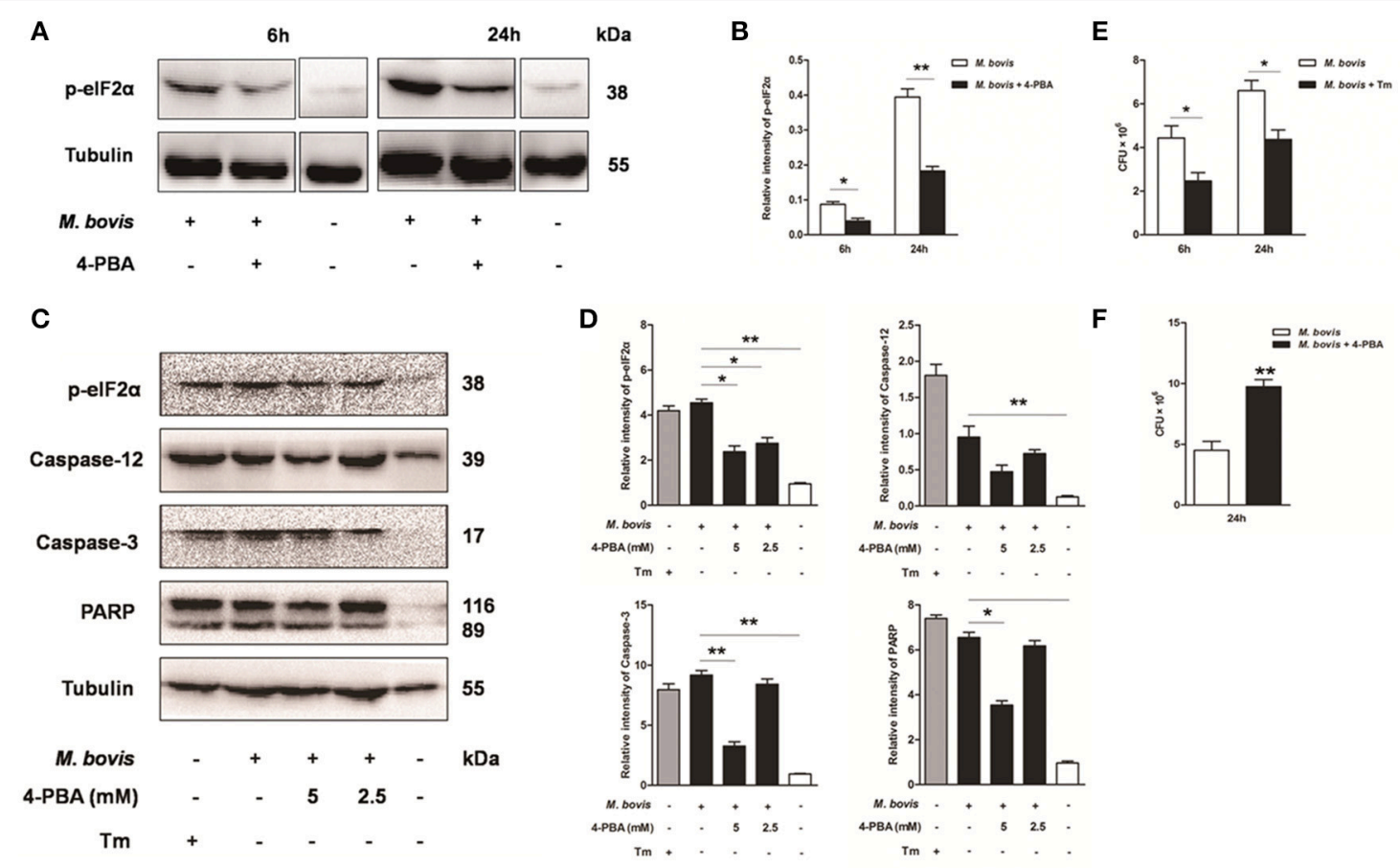

FIGURE 3 | Effect of ER stress mediated apoptosis on intracellular survival of $\boldsymbol{M}$. bovis. (A-D) 4-PBA was treated before M. bovis infection in Raw 264.7 cells and total cell lysates were subjected to Western blot for phosphorylation of elF2 $\alpha$, cleaved caspase-12, and caspase-3 (p17 specific) and PARP. Bands corresponding to each protein were quantified, and the intensities of each protein were normalized to the intensity of tubulin. (E) Quantification of intracellular survival of $M$. bovis in Raw 264.7 cells pretreated for $3 \mathrm{~h}$ with tunicamycin $(5 \mu \mathrm{g} / \mathrm{ml})$. Cells were harvested at 6 and $24 \mathrm{~h}$ post infection with $M$. bovis and bacteria number was determined by CFU counting. (F) Quantification of intracellular survival of $M$. bovis in Raw 264.7 cells pretreated for $3 \mathrm{~h}$ with $4-\mathrm{PBA}$ (5 mM). Cells were harvested at $24 \mathrm{~h}$ post infection with $M$. bovis and bacterial numbers were determined. Data are representative of at least three independent experiments, each performed in triplicate with similar results. The asterisks indicate significant differences compared with untreated cells $\left({ }^{\star} P<0.05,{ }^{* \star} P<0.01\right)$.

We further confirmed that the activation of IRF3 during $M$. bovis infection by immunofluorescence. IRF3 was significantly detected in the nucleus, while no fluorescent clusters were found in uninfected control cells (Figure 4E). Raw 264.7 cells were treated with $5 \mathrm{mM} 4$-PBA before $M$. bovis infection to verify that phosphorylation of IRF3 was mediated by ER stress. As shown in Figures 4C-E, phosphorylation and nuclear translocation of IRF3 were reduced. These results indicate that ER stress results in the phosphorylation and the nuclear translocation of IRF3 during M. bovis infection.

\section{Activation of IRF3 Requires Mobilization of STING and TBK1}

As an adaptor, STING predominantly resides in the ER. Recent studies have shown that calcium mobilization is involved in ER stress, which causes the translocation of STING and subsequently activates the downstream molecule TBK1 in the cytoplasm. TBK1 is an upstream serine/threonine kinase that plays an essential role in phosphorylating IRF3 (Liu et al., 2012; Tanaka and Chen, 2012; Petrasek et al., 2013). To verify whether the activation of IRF3 was associated with TBK1, Raw 264.7 cells were treated with the potent and specific TBK1 inhibitor BX-795 before $M$. bovis infection. Levels of phosphorylation of IRF3 decreased in a dose-dependent manner in response to 5 and $10 \mu \mathrm{M}$ BX-795 (Figures 4A,B).
To investigate whether STING-TBK1 pathway was involved in $M$. bovis infection, phosphorylation of TBK1 was studied in a time-course macrophage infection model. We found that phosphorylation of TBK1 increased in $24 \mathrm{~h}$ pi and decreased by $48 \mathrm{~h}$ pi, but no difference was identified at $6 \mathrm{~h} \mathrm{pi}$, compared to uninfected control cells (Figures 5A,B). Next, we studied whether the activation of TBK1 was associated with ER stress. Raw 264.7 cells treated with 4-PBA prior to $M$. bovis infection showed that the phosphorylation of TBK1 was significantly attenuated by the treatment with $4-\mathrm{PBA}$ at $24 \mathrm{~h}$ pi (Figures 5C,D).

To determine whether the translocation of STING caused the phosphorylation of TBK1, we interrogated the interaction between STING and TBK1. By immunofluorescence microscopy, a striking co-localization of STING and TBK1 was identified, with association into larger clusters around the nucleus at $24 \mathrm{~h}$ pi. But this co-localization was abrogated when cells were pretreated by 4 -PBA (Figures 5E,F). Together, these data suggest that the activation of IRF3 requires the mobilization of STING and TBK1, mediated by ER stress during M. bovis infection.

\section{IRF3 Is Required for Mitochondrial Damage during $M$. bovis Infection}

It is well-known that IRF3 plays a critical role in innate immunity by regulating inflammation and type-I interferons (IFNs) (Opitz 
A

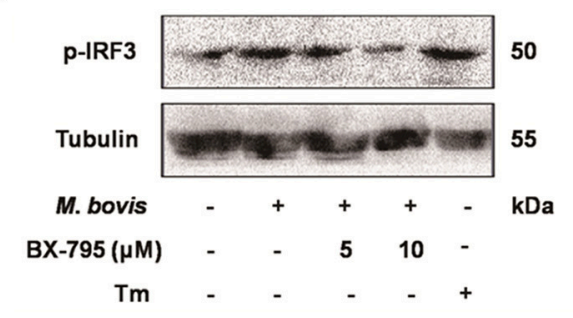

C

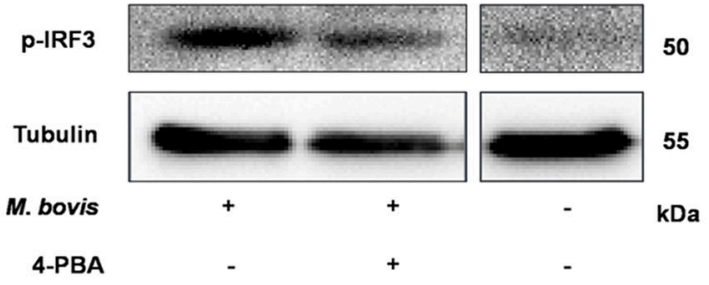

E

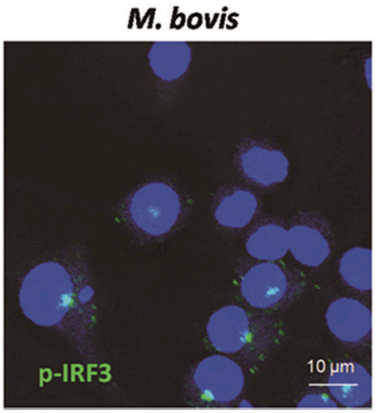

M. bovis + 4-PBA

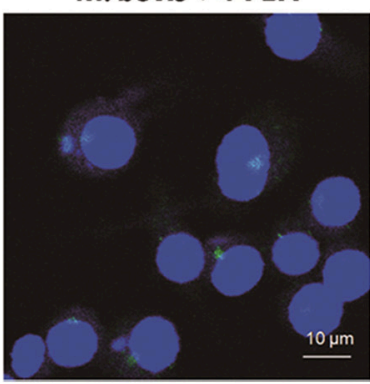

B

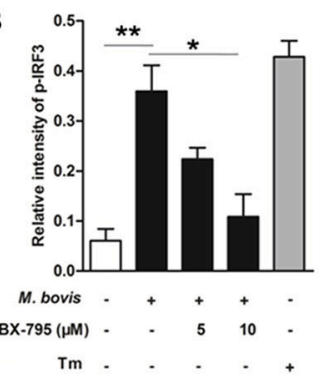

D

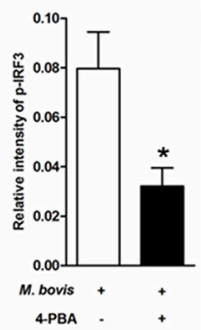

Control

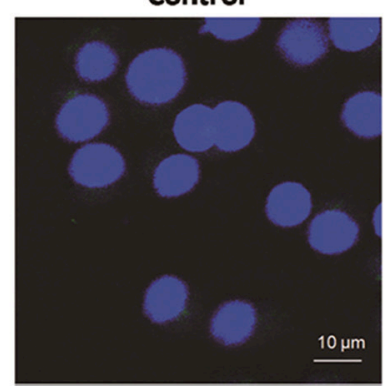

FIGURE 4 | ER stress results in the phosphorylation and nuclear translocation of IRF3. (A-D) BX-795 and 4-PBA were respectively treated before M. bovis infection in Raw 264.7 cells and total cell lysates were subjected to Western blot for phosphorylation of IRF3. Bands corresponding to each protein were quantified, and the intensities of each protein were normalized to the intensity of tubulin. The asterisks indicate significant differences compared with untreated cells ${ }^{\star} P<0.05$, $\left.{ }^{\star \star} P<0.01\right)$. (E) 4-PBA was treated before M. bovis infection in Raw 264.7 cells. Fixed cells were incubated with anti-IRF3 monoclonal antibody followed by FITC-conjugated goat anti-rabbit antibody as the secondary antibody and visualized by immunofluorescence microscopy. Results are representative of three independent experiments, each performed in triplicate with similar results.

et al., 2006). Recent studies have identified that activated IRF3 binds cytosolic Bax, which results in Bax translocation to the mitochondria and initiation of the intrinsic apoptotic pathway in response to several types of stimuli such as viruses and alcohol (Chattopadhyay et al., 2010; Petrasek et al., 2013). To verify if this pathway was conserved in $M$. bovis infection, we first assessed mitochondrial function by JC-1 mitochondrial transmembrane potential $(\Delta \Psi \mathrm{m})$ assay (Figure 6A). After infection with M. bovis for $24 \mathrm{~h}$, green fluorescence (JC-1 monomer form) increased and red fluorescence (JC-1 aggregates form) decreased, indicating low $\Delta \Psi \mathrm{m}$ values and thus mitochondrial dysfunction compared against the negative control. Then we further tested the translocation of Bax and cytochrome c. We found that Bax was detected in isolated mitochondria and was reduced in the cytoplasm. In contrast, cytochrome $\mathrm{c}$ was released from mitochondria to the cytoplasm after $M$. bovis infection for $24 \mathrm{~h}$ (Figure 6B). To investigate if this process was associated with the phosphorylation of IRF3, Raw 264.7 cells were infected with
M. bovis after pre-treatment with BX-795. The translocation of Bax and the release of cytochrome $\mathrm{c}$ were significantly inhibited. Furthermore, we detected the interaction between IRF3 and Bax by co-immunoprecipitation. As shown in Figure 6C, the phosphorylated IRF3 bound to Bax during $M$. bovis infection. Collectively, these data demonstrate that $M$. bovis stimulated the phosphorylation of IRF3 in the cytoplasm, where IRF3 together with bax translocate into the mitochondria to activate mitochondrial outer membrane permeabilization (MOMP), leading to the release of cytochrome c subsequently damaging the organelle and cause apoptotic cell death.

\section{IRF3 also Causes Caspase-8 Activation and Controls Intracellular Survival of M. bovis}

Previous studies indicate that the extrinsic cell death regulators FADD and caspase- 8 are linked to IRF3 activation 
A

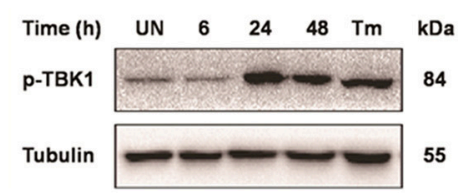

C

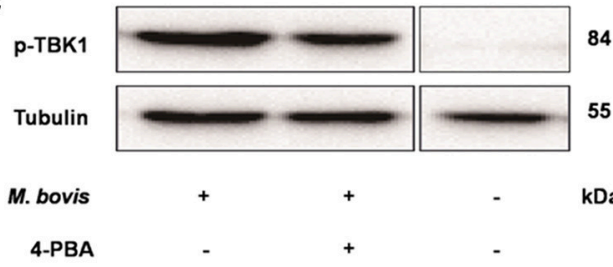

B
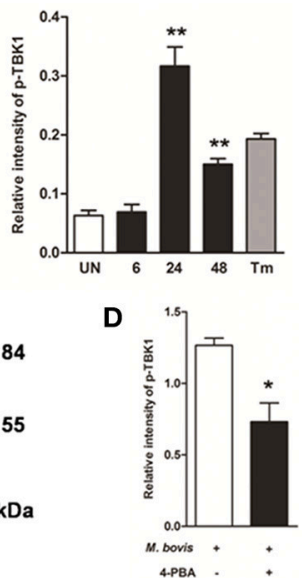

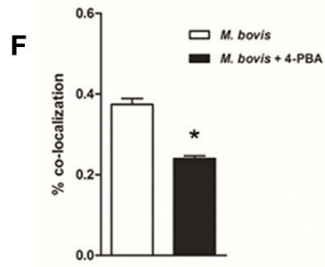

E Anti-STING
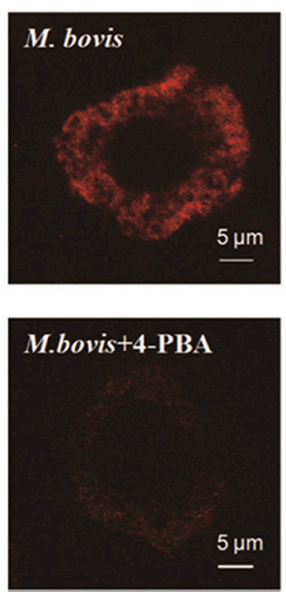

Anti-TBK1
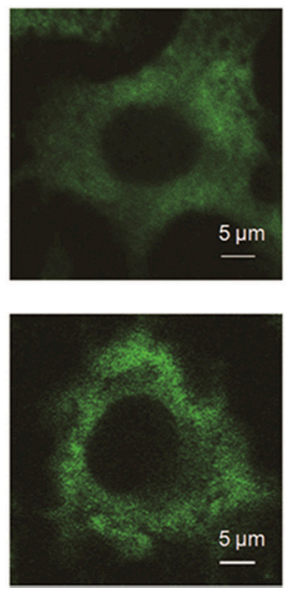

DAPI
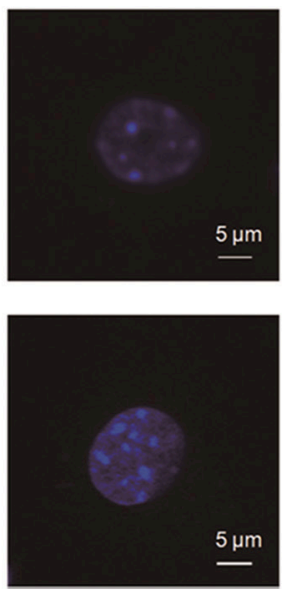

Merge
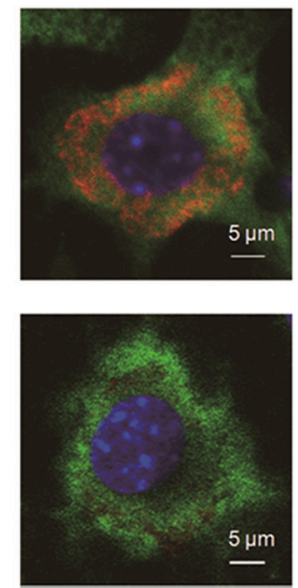

FIGURE 5 | Activation of IRF3 requires the mobilization of STING and TBK1. (A-D) Raw 264.7 cells were infected with M. bovis at an MOI of 10, and then incubated for $\mathbf{0}-48 \mathrm{~h}$. Total cell lysates were subjected to Western blot for phosphorylation of TBK1. Bands corresponding to each protein were quantified, and the intensities of each protein were normalized to the intensity of tubulin. (C,D) 4-PBA was treated before M. bovis infection in Raw 264.7 cells and total cell lysates were subjected to Western blot for phosphorylation of TBK1. (E,F) 4-PBA was treated before M. bovis infection in Raw 264.7 cells. Fixed cells were incubated with anti-STING antibody and anti-TBK1 antibody followed by fluorescein isothiocyanate (FITC)-conjugated donkey anti-goat (green) or Alexa Fluor-conjugated goat anti-rabbit (red). Results are representative of at least three independent experiments, each performed in triplicate with similar results. The asterisks indicate significant differences compared with untreated cells $\left({ }^{\star} P<0.05\right.$, $\left.{ }^{\star \star} P<0.01\right)$.

in hepatocyte apoptosis (Petrasek et al., 2013; Liu et al., 2015). However, this has not been studied in mycobacterial infection. Raw 264.7 cells treated with BX-795 before $M$. bovis infection were studied on this effect. Caspase- 8 and caspase-3 were decreased in a dose-dependent manner in response to BX-795 at $24 \mathrm{~h}$ pi (Figures 6D,E). Intracellular survival of $M$. bovis was sustained when BX-795 was used (Figure 6F). These findings show that the extrinsic cell death pathway may also be involved in IRF3-mediated apoptosis. And IRF3 can partly control intracellular survival of bacteria.

These data highlight that (i) M. bovis-induced apoptosis is mediated by ER stress and (ii) STING-TBK1-IRF3 pathway is activated by ER stress, which finally leads to apoptosis (Figure 7).

\section{DISCUSSION}

Mycobacteria are highly adapted to macrophages and have multiple mechanisms to resist the host immune responses (Liu et al., 2015). Apoptosis is considered as a crucial outcome for the host to control intracellular bacteria (Aguilo et al., 2013; Divangahi et al., 2013). Thus, it is necessary to understand the exact mechanisms of apoptosis regulation during mycobacterial infection.

Previous studies have identified that a low MOI infection of virulent Mtb leads to macrophage apoptosis, while a high MOI infection induces necrosis (Lee et al., 2006, 2009; Lim et al., 2011). In preliminary experiments, we found that Raw 264.7 cells infected with $M$. bovis at an MOI of 10 induced more apoptosis than at MOI of 1, 5 with few cells showing signs of necrosis. 
A

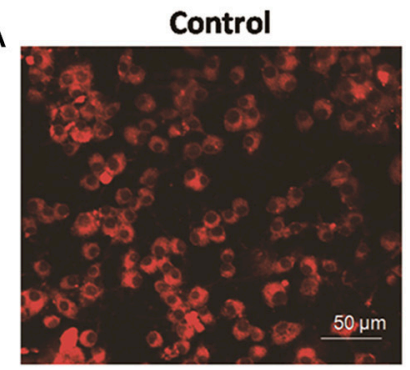

B

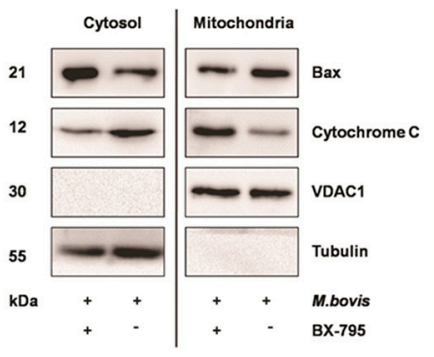

C
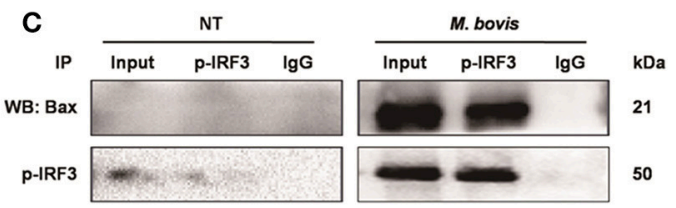

CCCP

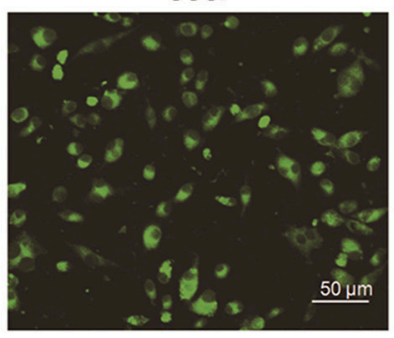

D

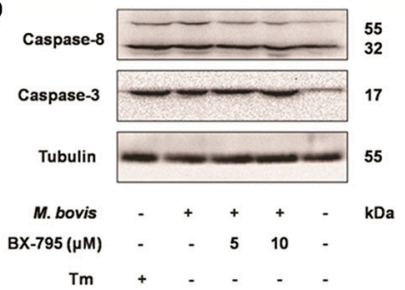

E

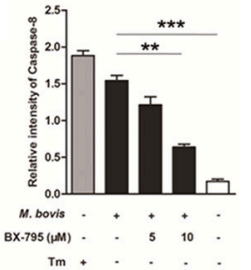

M. bovis

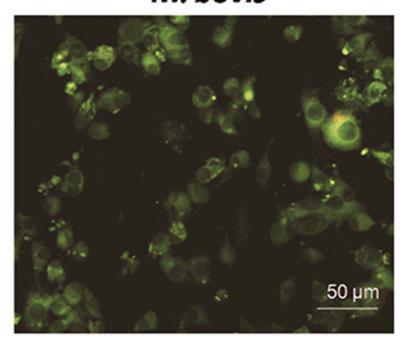

F
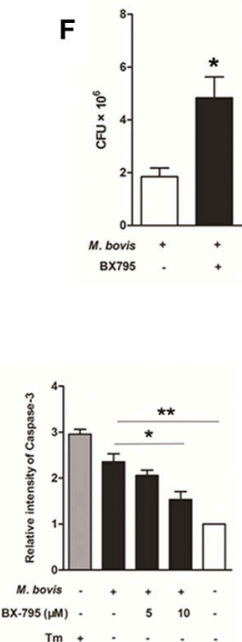

FIGURE 6 | IRF3 causes the mitochondrial damage and caspase-8 activation. Raw 264.7 cells were untreated or treated with $\mathrm{M}$. bovis at an MOI of 10 for $24 \mathrm{~h}$. (A) Mitochondrial transmembrane potential $(\Delta \Psi \mathrm{m})$ is measured using JC-1 as the fluorescent marker. The JC-1-aggregate form, indicating normal $\Delta \Psi \mathrm{m}$, appears red and the monomeric form, indicating low $\Delta \Psi \mathrm{m}$ (i.e., disrupted mitochondrial membrane), is green by confocal microscopy. Carbonyl cyanide m-chlorophenylhydrazone (CCCP), as a positive control for the mitochondrial damage. Scale bar $=50 \mu \mathrm{m}$. (B) Mitochondrial fractions were isolated from whole cell lysates from Raw 264.7 cells. Mitochondrial fractions and supernatant were immunoblotted for the indicated proteins. Left and right parts represent the same membrane. (C) Samples were immunoprecipitated with control IgG or anti-phospho-IRF3. Whole-cell lysate (input) or immunoprecipitations were resolved by SDS-PAGE and immunoblotted with anti-phospho-IRF3 or Bax. (D) BX-795 was treated before M. bovis infection in Raw 264.7 cells and total cell lysates were subjected to Western blot for cleaved caspase-3 (p17 specific) and caspase-8. (E) Bands corresponding to each protein were quantified, and the intensities of each protein were normalized to the intensity of tubulin. (F) Quantification of intracellular survival of M. bovis in Raw 264.7 cells pretreated for $3 \mathrm{~h}$ with BX-795 (10 $\mu \mathrm{M})$. Cells were harvested at $24 \mathrm{~h}$ post infection with $M$. bovis and bacteria number was determined by CFU counting. Data are representative of at least three independent experiments, each performed in triplicate with similar results. The asterisks indicate significant differences compared with untreated cells $\left({ }^{\star} P<0.05\right.$, ${ }^{\star \star} P<0.01$, $\left.{ }^{\star \star \star} P<0.001\right)$.

Thus, an MOI of 10 was chosen for these studies. This difference in effective infective dose is likely to be defined by the infecting strain genotype.

In the present study, apoptosis was verified to be caspase dependent. Caspase-3, caspase-9, and caspase-12 were activated by $M$. bovis infection. Caspase- 12 is localized to the ER membrane and can be activated by the calcium activated protease calpain, or separating from TRAF2 or the translocation of caspase-7 upon ER stress. Activated caspase-12 translocates to the cytosol, where it directly cleaves pro-caspase- 9 that next cleaves caspase-3. This cascade is not dependent on either mitochondria or death receptor pathway (Nakagawa and Yuan, 2000; Yoneda et al., 2001; Szegezdi et al., 2003).

Recent studies suggested that $M$. bovis infection results in loss of $\mathrm{Ca}^{2+}$ from the ER and an increase in the intracellular redox state which results in accumulation of unfolded or misfolded proteins in the ER resulting in ER stress (Choi et al., 2010;
Lim et al., 2015). In the present study, we first observed the morphology of ER by TEM in order to determine whether ER stress was involved in $M$. bovis infection. The expansion of the ER compartment in early post infection time points prompted us to continue to test the ER stress-related indicators. The ratio of the splicing of XBP-1 mRNA was increased in a timedependent manner at $9 \mathrm{~h}$ pi and gradually decreased by $48 \mathrm{~h}$ pi. The phosphorylation of eIF $2 \alpha$ was increased in a time-dependent manner in $24 \mathrm{~h}$. These results indicate that the phosphorylated IRE1 $\alpha$ mainly activate the downstream transcription factor XBP1 in early post infection. Then XBP-1s likely binds to the UPR element (UPRE) and to the ERS-response elements I and II (ERSE-I and ERSE-II) in the promoter regions of target genes inducing the expression of a large number of other related genes to reduce or abrogate ER stress and restore ER homeostasis (Cao and Kaufman, 2012). Unlike the protective role of IRE1 $\alpha$ pathway in the early stages of infection, the PERK pathway plays 


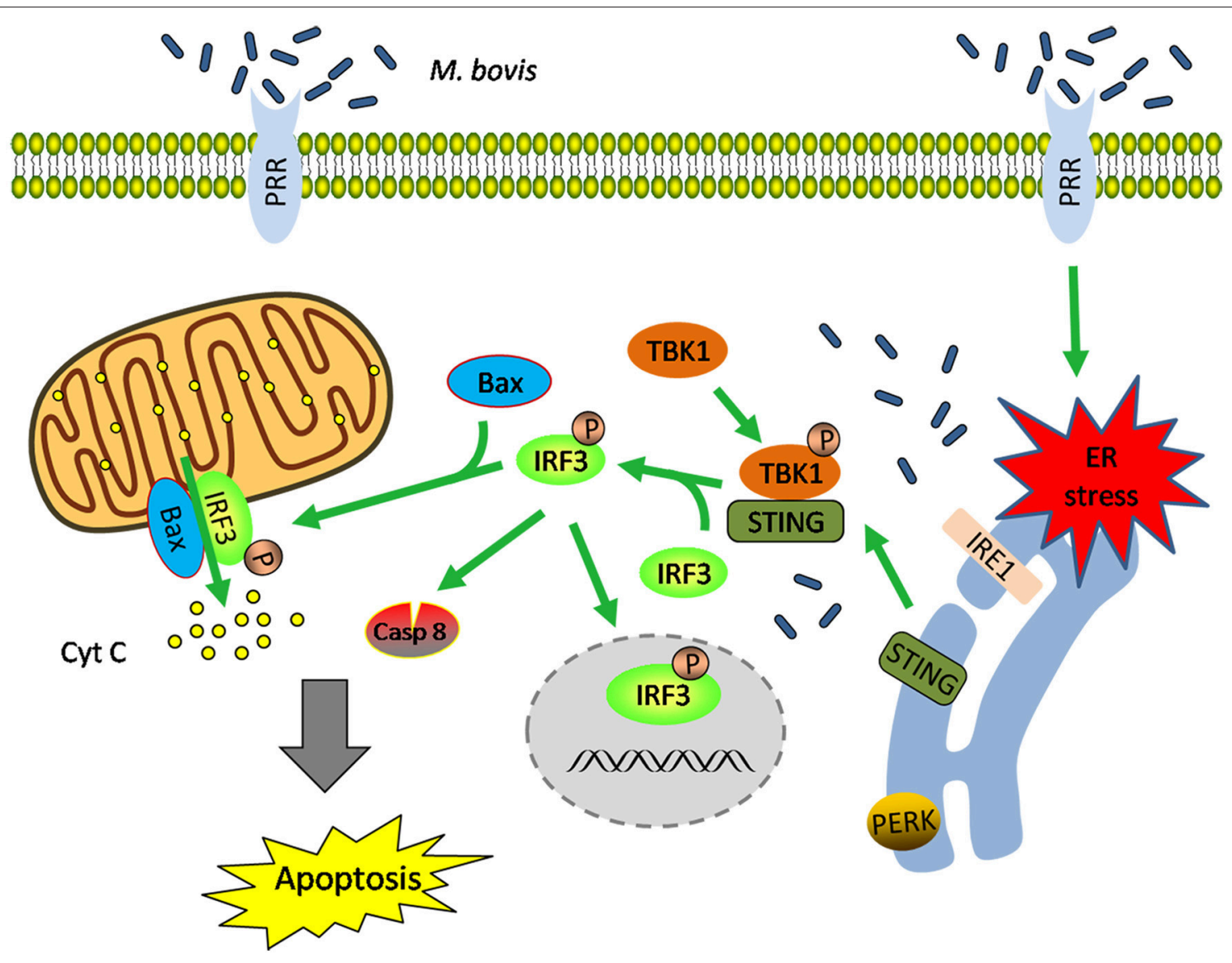

FIGURE 7 | Schematic representation of ER stress-mediated apoptosis via the activation of IRF3 following $M$. bovis stimulation. $M$. bovis induced ER stress in macrophages triggers the translocation of STING, which facilitates phosphorylation of IRF3 by the downstream phosphorylated TBK1. Subsequently, (i) phosphorylated IRF3 translocates to the nucleus to induce the transcription of putative apoptosis-related factors. (ii) phosphorylated IRF3 associates with Bax and triggers mitochondrial pathway of macrophages apoptosis. (iii) phosphorylated IRF3 activates the downstream caspase-8. All these three pathways finally lead to apoptosis.

an apoptotic role in the late stages. Similarly, an early onset of XBP-1 splicing and a slow translation of ATF4 for cell survival were observed by imaging the single cell response to ER stress (Walter et al., 2015). We also confirmed this apoptosis caused by ER stress is beneficial in controlling the number of intracellular bacteria by pre-treatment with the specific agonist and inhibitor of ER stress.

Emerging evidence suggests that IRF3, a transcription factor regulating innate immune responses, was involved in ER stressmediated apoptosis (Liu et al., 2012; Petrasek et al., 2013; Jin et al., 2015). However, no reports have shown that this participation of IRF3 upon ER stress links to mycobacterial infections. In general, inactivated IRF3 exists in the cytoplasm. Once ER stress occurs, IRF3 will autophosphorylate and translocate to the nucleus to induce transcription of IFN- $\beta$, inflammatory cytokines and apoptosis-related factors (O'Connell et al., 2004; Liu et al., 2012). Furthermore, we detected the phosphorylation and translocation of IRF3 in $24 \mathrm{~h}$. We then confirmed the activation of IRF3 was mediated by ER stress by the pre-treatment with 4-PBA. Next, the activation mechanisms of IRF3 during $M$. bovis infection needed to be clarified. One recent study indicated that different ER stressors use distinct mechanisms to activate IRF3, such as Tm is related to the activation of ATF6 processing, while thapsigargin is linked to the calcium mobilization and the requirement of STING and TBK1 (Liu et al., 2012). Another study has shown that cytosolic dsDNA released from mycobacteria via the ESX-1 secretion system could cause the innate immune response by cGAS-STING pathway (Collins et al., 2015). And also mycobacterial infection could result in loss of $\mathrm{Ca}^{2+}$ from the ER (Choi et al., 2010; Lim et al., 2015). Thus, we focused on and hypothesized that the mechanism of IRF3 activation is related to the STING-TBK1 pathway upon ER stress during M. bovis infection. STING is an ER-resident protein, harboring three functional domains: A cytoplasmic C-terminal tail, a central globular domain, and four putative $\mathrm{N}$-terminal transmembrane motifs which anchor STING to the ER (Ishikawa and Barber, 2008; Sun et al., 2009). STING is not only highly expressed in immune-related cells, but also plays an important role in function as its evolutionary conservation, such as human STING and mouse STING share approximate $68 \%$ sequence identity 
and $81 \%$ similarity at the amino acid level, especially at the C-terminal region (Zhong et al., 2008; Sun et al., 2009). STING undergoes phosphorylation, ubiquitination and translocates to the cytoplasm upon ER stress, which serves as a platform for the recruitment and auto-phosphorylation of the serine-threonine kinase TBK1 and subsequently phosphorylates and activates the transcription factor IRF3 (Liu et al., 2012). In our studies, the phosphorylation of TBK1 and the co-localization of STING and TBK1 were both observed after $M$. bovis stimulation. STING and TBK1 pathway activation was associated with ER stress by the inhibitor 4-PBA. We discovered that STING-TBK1-IRF3 pathway can be activated by ER stress during $M$. bovis infection, but which specific pathway of ER stress mediated the activation of STING-TBK1-IRF3 pathway remains to be determined.

Finally, we investigated how IRF3 induced apoptosis upon ER stress during $M$. bovis infection. Numerous studies have addressed three major mechanisms of IRF3-mediated apoptosis: (i) homodimers of IRF3 induce transcription of the BH3only proteins Noxa and Puma, which antagonizes pro-survival $\mathrm{Bcl}-2$ proteins such as $\mathrm{Mcl}-1$ and $\mathrm{Bcl}-\mathrm{xL}$ to activate Bax and Bak and to damage mitochondria, leading to the release of cytochrome $\mathrm{c}$ which causes apoptosis. (ii) activated-IRF3 binds cytosolic Bax through a $\mathrm{BH} 3$-like domain to the mitochondria to induce the release of cytochrome $\mathrm{c}$ which causes apoptosis. (iii) the recruitment of FADD and caspase- 8 results in caspase8 activation, which cleaves Bid to antagonize pro-survival Bcl-2 family members allowing Bax and Bak to induce cell death (Vince and Tschopp, 2010). In the present study, we found the translocation of Bax and the release of cytochrome c. The interaction of IRF3 and Bax was observed by coimmunoprecipitation. Splicing of caspase- 8 and caspase- 3 was decreased in a dose-dependent manner in response to BX795. Previous studies show that TBK1 participates in autophagy to control mycobacterium survival (Pilli et al., 2012). But our results document that apoptosis mediated by IRF3 partly control intracellular bacteria which is also in contrast to the results presented in a previous study that showed IRF3-/-

\section{REFERENCES}

Aguilo, N., Marinova, D., Martin, C., and Pardo, J. (2013). ESX-1-induced apoptosis during mycobacterial infection: to be or not to be, that is the question. Front. Cell Infect. Microbiol. 3:88. doi: 10.3389/fcimb.2013.00088

Aldwell, F. E., Wedlock, D. N., and Buddle, B. M. (1996). Bacterial metabolism, cytokine mRNA transcription and viability of bovine alveolar macrophages infected with Mycobacterium bovis BCG or virulent M. bovis. Immunol. Cell Biol. 74, 45-51. doi: 10.1038/icb.1996.6

Arap, M. A., Lahdenranta, J., Mintz, P. J., Hajitou, A., Sarkis, A. S., Arap, W., et al. (2004). Cell surface expression of the stress response chaperone GRP78 enables tumor targeting by circulating ligands. Cancer Cell 6, 275-284. doi: 10.1016/j.ccr.2004.08.018

Cao, S. S., and Kaufman, R. J. (2012). Unfolded protein response. Curr. Biol. 22, R622-R626. doi: 10.1016/j.cub.2012.07.004

Chattopadhyay, S., Marques, J. T., Yamashita, M., Peters, K. L., Smith, K., Desai, A., et al. (2010). Viral apoptosis is induced by IRF-3-mediated activation of Bax. EMBO J. 29, 1762-1773. doi: 10.1038/emboj.2010.50

Choi, H. H., Shin, D. M., Kang, G., Kim, K. H., Park, J. B., Hur, G. M., et al. (2010). Endoplasmic reticulum stress response is involved in Mycobacterium mice are better at controlling mycobacteria than wild type mice (Manzanillo et al., 2012). It is likely that our cell models provide a more simplistic albeit pristine look at macrophagemycobacterium interactions compared to a genetically modified mouse model. Alternately, since our study looked at $M$. bovis vs. $M t b$ by Manzanillo et al, pathogen specific responses, that may explain the differences in findings, cannot be ruled out. Taken together, our results identify a new and essential role of STING-TBK1-IRF3 pathway mediating a cross-talk between ER stress and apoptosis during M. bovis infection. To our knowledge, this is the first study to demonstrate a relationship between the activation of IRF3 and apoptosis in M. bovis infection. The data indicates that not only ER stress, but also IRF3 induced by ER stress can effectively control intracellular bacteria, which affords an alternate approach for the treatment of TB.

\section{AUTHOR CONTRIBUTIONS}

$\mathrm{YC}$ and $\mathrm{XZ}$ designed the study; YC performed experiments; YC, CL, WY, ZS, SS, and PB analyzed the data; YC drafted the manuscript; DZ, LY, and XZ contributed to conduct of the laboratory work and critical review of the manuscript. All authors contributed to read and approved the final version.

\section{ACKNOWLEDGMENTS}

We thank Dr. Jin Wang, Dr. Ruichao Yue, Dr. Chaosi Li, Dr. Guangyu Cheng for their valuable suggestions and critical reading of this manuscript. This work was supported by the MoSTRCUK international cooperation project (Project No. 2013DFG32500), National Natural Science Foundation of China (Project No. 31572487), Funding of State Key Lab of Agrobiotechnology (Project No. 2012SKLAB06-14). 2015 CAU Foreign Experts Major Projects (Project No: 2012z018). High-end Foreign Experts Recruitment Program (Project No: GDW20151100036).

tuberculosis protein ESAT-6-mediated apoptosis. FEBS Lett. 584, 2445-2454. doi: 10.1016/j.febslet.2010.04.050

Choi, J.-A., Lim, Y.-J., Cho, S.-N., Lee, J.-H., Jeong, J.-A., Kim, E.-J., et al. (2013). Mycobacterial HBHA induces endoplasmic reticulum stress-mediated apoptosis through the generation of reactive oxygen species and cytosolic $\mathrm{Ca}^{2+}$ in murine macrophage RAW 264.7 cells. Cell Death Dis. 4:e957. doi: $10.1038 /$ cddis.2013.489

Collins, A. C., Cai, H. C., Li, T., Franco, L. H., Li, X. D., Nair, V. R., et al. (2015). Cyclic GMP-AMP synthase is an innate immune DNA sensor for Mycobacterium tuberculosis. Cell Host Microbe 17, 820-828. doi: 10.1016/j.chom.2015.05.005

Cui, Y., Zhao, D., Barrow, P. A., and Zhou, X. (2016). The endoplasmic reticulum stress response: a link with tuberculosis? Tuberculosis 97, 52-56. doi: 10.1016/j.tube.2015.12.009

Divangahi, M., Behar, S. M., and Remold, H. (2013). Dying to live: how the death modality of the infected macrophage modulates immunity to tuberculosis. $A d v$. Exp. Med. Biol. 783, 103-120. doi: 10.1007/978-1-4614-6111-1_6

Hetz, C. (2012). The unfolded protein response: controlling cell fate decisions under ER stress and beyond. Nat. Rev. Mol. Cell Biol. 13, 89-102. doi: $10.1038 / \mathrm{nrm} 3270$ 
Ishikawa, H., and Barber, G. N. (2008). STING is an endoplasmic reticulum adaptor that facilitates innate immune signalling. Nature 455:U674. doi: $10.1038 /$ nature 07317

Jin, L., Wang, K., Liu, H., Chen, T., Yang, Y., Ma, X., et al. (2015). Genomewide histone $\mathrm{H} 3$ lysine 9 acetylation profiling in $\mathrm{CD} 4+\mathrm{T}$ cells revealed endoplasmic reticulum stress deficiency in patients with acute-on-chronic liver failure. Scand. J. Immunol. 82, 452-459. doi: 10.1111/sji.12341

Lee, J., Hartman, M., and Kornfeld, H. (2009). Macrophage apoptosis in tuberculosis. Yonsei Med. J. 50, 1-11. doi: 10.3349/ymj.2009.50.1.1

Lee, J., Remold, H. G., Leong, M. H., and Kornfeld, H. (2006). Macrophage apoptosis in response to high intracellular burden of Mycobacterium tuberculosis is mediated by a novel caspase-independent pathway. J. Immunol. 176, 4267-4274. doi: 10.4049/jimmunol.176.7.4267

Lim, Y. J., Choi, H. H., Choi, J. A., Jeong, J. A., Cho, S. N., Lee, J. H., et al. (2013). Mycobacterium kansasii-induced death of murine macrophages involves endoplasmic reticulum stress responses mediated by reactive oxygen species generation or calpain activation. Apoptosis 18, 150-159. doi: 10.1007/s10495-012-0792-4

Lim, Y. J., Choi, J. A., Choi, H. H., Cho, S. N., Kim, H. J., Jo, E. K., et al. (2011). Endoplasmic reticulum stress pathway-mediated apoptosis in macrophages contributes to the survival of Mycobacterium tuberculosis. PLoS ONE 6:e28531. doi: 10.1371/journal.pone.0028531

Lim, Y. J., Choi, J. A., Lee, J. H., Choi, C. H., Kim, H. J., and Song, C. H. (2015), Mycobacterium tuberculosis $38-\mathrm{kDa}$ antigen induces endoplasmic reticulum stress-mediated apoptosis via toll-like receptor 2/4. Apoptosis 20, 358-370. doi: 10.1007/s10495-014-1080-2

Liu, M. Q., Li, W., Xiang, X. H., and Xie, J. P. (2015). Mycobacterium tuberculosis effectors interfering host apoptosis signaling. Apoptosis 20, 883-891. doi: 10.1007/s10495-015-1115-3

Liu, Y. P., Zeng, L., Tian, A., Bomkamp, A., Rivera, D., Gutman, D., et al. (2012). Endoplasmic reticulum stress regulates the innate immunity critical transcription factor IRF3. J. Immunol. 189, 4630-4639. doi: $10.4049 /$ jimmunol.1102737

Majumdar, T., Chattopadhyay, S., Ozhegov, E., Dhar, J., Goswami, R., Sen, G. C., et al. (2015). Induction of interferon-stimulated genes by IRF3 promotes replication of Toxoplasma gondii. PLoS Pathog. 11:e1004779. doi: 10.1371/journal.ppat.1004779

Manzanillo, P. S., Shiloh, M. U., Portnoy, D. A., and Cox, J. S. (2012). Mycobacterium Tuberculosis activates the DNA-dependent cytosolic surveillance pathway within macrophages. Cell Host Microbe 11, 469-480. doi: 10.1016/j.chom.2012.03.007

Nakagawa, T., and Yuan, J. Y. (2000). Cross-talk between two cysteine protease families: activation of caspase-12 by calpain in apoptosis. J. Cell Biol. 150, 887-894. doi: 10.1083/jcb.150.4.887

O'Connell, R. M., Saha, S. K., Vaidya, S. A., Bruhn, K. W., Miranda, G. A., Zarnegar, B., et al. (2004). Type I interferon production enhances susceptibility to Listeria monocytogenes infection. J. Exp. Med. 200, 437-445. doi: 10.1084/jem.20040712

Opitz, B., Vinzing, M., van Laak, V., Schmeck, B., Heine, G., Günther, S., et al. (2006). Legionella pneumophila induces IFN $\beta$ in lung epithelial cells via IPS-1 and IRF3, which also control bacterial replication. J. Bio. Chem. 281, 36173-36179. doi: 10.1074/jbc.M604638200

Petrasek, J., Iracheta-Vellve, A., Csak, T., Satishchandran, A., Kodys, K., KurtJones, E. A., et al. (2013). STING-IRF3 pathway links endoplasmic reticulum stress with hepatocyte apoptosis in early alcoholic liver disease. Proc. Natl. Acad. Sci. U.S.A. 110, 16544-16549. doi: 10.1073/pnas.1308331110

Pilli, M., Arko-Mensah, J., Ponpuak, M., Roberts, E., Master, S., Mandell, M. A., et al. (2012). TBK-1 promotes autophagy-mediated antimicrobial defense by controlling autophagosome maturation. Immunity 37, 223-234. doi: 10.1016/j.immuni.2012.04.015

Rasheva, V. I., and Domingos, P. M. (2009). Cellular responses to endoplasmic reticulum stress and apoptosis. Apoptosis 14, 996-1007. doi: 10.1007/s10495-009-0341-y

Rodrigues, M. F., Alves, C. C., Figueiredo, B. B., Rezende, A. B., Wohlres-Viana, S., Silva, V. L., et al. (2013). Tumour necrosis factor receptors and apoptosis of alveolar macrophages during early infection with attenuated and virulent Mycobacterium bovis. Immunology 139, 503-512. doi: 10.1111/imm.12097

Ron, D., and Walter, P. (2007). Signal integration in the endoplasmic reticulum unfolded protein response. Nat. Rev. Mol. Cell Biol. 8, 519-529. doi: $10.1038 / \mathrm{nrm} 2199$
Sano, R., and Reed, J. C. (2013). ER stress-induced cell death mechanisms. Biochim. Biophys. Acta 1833, 3460-3470. doi: 10.1016/j.bbamcr.2013. 06.028

Saquib, N. M., Jamwal, S., Midha, M. K., Verma, H. N., and Manivel, V. (2015). Quantitative proteomics and lipidomics analysis of endoplasmic reticulum of macrophage infected with Mycobacterium tuberculosis. Int. J. Proteomics 2015:270438. doi: 10.1155/2015/270438

Seimon, T. A., Kim, M. J., Blumenthal, A., Koo, J., Ehrt, S., Wainwright, H., et al. (2010). Induction of ER stress in macrophages of tuberculosis granulomas. PLoS ONE 5:e12772. doi: 10.1371/journal.pone.0012772

Sohn, H., Kim, J. S., Shin, S. J., Kim, K., Won, C. J., Kim, W. S., et al. (2011). Targeting of Mycobacterium tuberculosis heparin-binding hemagglutinin to mitochondria in macrophages. PLoS Pathog. 7:e1002435. doi: 10.1371/journal.ppat. 1002435

Srinivasan, L., Ahlbrand, S., and Briken, V. (2014). Interaction of Mycobacterium tuberculosis with host cell death pathways. Cold Spring Harb. Perspect. Med. 4:a022459. doi: 10.1101/cshperspect.a022459

Sun, W. X., Li, Y., Chen, L., Chen, H. H., You, F. P., Zhou, X., et al. (2009). ERIS, an endoplasmic reticulum IFN stimulator, activates innate immune signaling through dimerization. Proc. Natl. Acad. Sci. U.S.A. 106, 8653-8658. doi: 10.1073/pnas.0900850106

Szegezdi, E., Fitzgerald, U., and Samali, A. (2003). Caspase-12 and ER-stressmediated apoptosis - the story so far. Ann. N. Y. Acad. Sci. 1010, 186-194. doi: $10.1196 /$ annals. 1299.032

Tabas, I., and Ron, D. (2011). Integrating the mechanisms of apoptosis induced by endoplasmic reticulum stress. Nat. Cell Biol. 13, 184-190. doi: $10.1038 /$ ncb0311-184

Tanaka, Y., and Chen, Z. J. (2012). STING specifies IRF3 phosphorylation by TBK1 in the cytosolic DNA signaling pathway. Sci. Signal. 5:ra20. doi: 10.1126/scisignal.2002521

Verfaillie, T., Rubio, N., Garg, A. D., Bultynck, G., Rizzuto, R., Decuypere, J. P., et al. (2012). PERK is required at the ER-mitochondrial contact sites to convey apoptosis after ROS-based ER stress. Cell Death Differ. 19, 1880-1891. doi: $10.1038 / \mathrm{cdd} .2012 .74$

Vince, J. E., and Tschopp, J. (2010). IRF-3 partners Bax in a viral-induced dance macarbre. EMBO J. 29, 1627-1628. doi: 10.1038/emboj.2010.79

Walter, F., Schmid, J., Dussmann, H., Concannon, C. G., and Prehn, J. H. M. (2015). Imaging of single cell responses to ER stress indicates that the relative dynamics of IRE1/XBP1 and PERK/ATF4 signalling rather than a switch between signalling branches determine cell survival. Cell Death Differ. 22, 1502-1516. doi: 10.1038/cdd.2014.241

Waters, W. R., and Palmer, M. V. (2015). Mycobacterium bovis infection of cattle and white-tailed deer: translational research of relevance to human tuberculosis. ILAR J. 56, 26-43. doi: 10.1093/ilar/ilv001

WHO (2014). Multi Drug Resistant TB 2014 Update. WHO.

Xu, G. H., Wang, J., Gao, G. F., and Liu, C. H. (2014). Insights into battles between Mycobacterium tuberculosis and macrophages. Protein Cell 5, 728-736. doi: 10.1007/s13238-014-0077-5

Yoneda, T., Imaizumi, K., Oono, K., Yui, D., Gomi, F., Katayama, T., et al. (2001). Activation of caspase-12, an endoplastic reticulum (ER) resident caspase, through tumor necrosis factor receptor-associated factor 2-dependent mechanism in response to the ER stress. J. Biol. Chem. 276, 13935-13940. doi: 10.1074/jbc.M010677200

Zhong, B., Yang, Y., Li, S., Wang, Y. Y., Li, Y., Diao, F. C., et al. (2008). The adaptor protein MITA links virus-sensing receptors to IRF3 transcription factor activation. Immunity 29, 538-550. doi: 10.1016/j.immuni.2008. 09.003

Conflict of Interest Statement: The authors declare that the research was conducted in the absence of any commercial or financial relationships that could be construed as a potential conflict of interest.

Copyright (c) 2016 Cui, Zhao, Sreevatsan, Liu, Yang, Song, Yang, Barrow and Zhou. This is an open-access article distributed under the terms of the Creative Commons Attribution License (CC BY). The use, distribution or reproduction in other forums is permitted, provided the original author(s) or licensor are credited and that the original publication in this journal is cited, in accordance with accepted academic practice. No use, distribution or reproduction is permitted which does not comply with these terms. 